\title{
Prof. Dr. Mathias Langer - Präsident des 94. Deutschen Röntgenkongresses (29. Mai bis 01. Juni 2013 im CCH in Hamburg)
}

Der diesjährige Präsident des 94. Deutschen Röntgenkongresses wird eine längere Anreise haben. Denn seit Juli 1992 ist Prof. Langer Ordinarius und Ärztlicher Direktor der Abteilung für Röntgendiagnostik der Radiologischen Klinik am Universitätsklinikum Freiburg an der AlbertLudwigs-Universität.

Geboren 1952 in Frankfurt am Main studierte er von 1970 bis 1976 Medizin an den Universitäten Straßburg und Köln. 1976 legte Prof. Langer zudem das amerikanische medizinische Staatsexamen (ECFMG) ab. Im Bereich physiologische Chemie erhielt er von der Deutschen Forschungsgemeinschaft ein Forschungsstipendium. Im Anschluss daran, ab 1978, erhielt Prof. Langer seine Facharztausbildung zum Radiologen an der Universitätsklinik Ulm. Doch auch dort hielt es ihn nicht lange. Forschungsaufenthalte an der Harvard Medical School und der University of San Francisco Medical School folgten. 1983 erhielt Prof. Langer die Anerkennung als Radiologie und 1991 zusätzlich die Teilgebietsbezeichnung Neuroradiologe. Seine Habilitation für das Fach Radiologe erlangte er 1985 an der Freien Universität Berlin und wurde 1989 zum Universitätsprofessor an der Freien Universität Berlin ernannt. Die Berufung auf den Lehrstuhl für Radiologie des Universitätsklinikums Jena folgte 1990. Nachfolgend nahm Prof. Langer 1992 die Berufung auf das Ordinariat in Freiburg an. Prof. Dr. Mathias Langer ist als Ärztlicher Direktor der Abteilung Röntgendiagnostik der Radiologischen Universitätsklinik an der Albert-Ludwigs-Universität seit 1992 in Freiburg tätig. Er war während dieser Zeit u.a. auch für 4 Jahre als Prorektor für Forschung und medizinische Angelegenheiten für die Gesamtuniversität aktiv.

Von den betriebswirtschaftlichen Instituten Chur und Köln erhielt Prof. Langer 2002 den Titel „Master of Hospital Business Administration“ (MBA) sowie die $\mathrm{Zu}$ satzbezeichnung „Diplom-Gesundheitsökonom“. Er zählt zu den führenden Fachärzten auf dem Gebiet der Computertomografie, mit einer besonderen Spezialisierung auf bösartige Tumoren, Kernspintomografien und Mammografien.

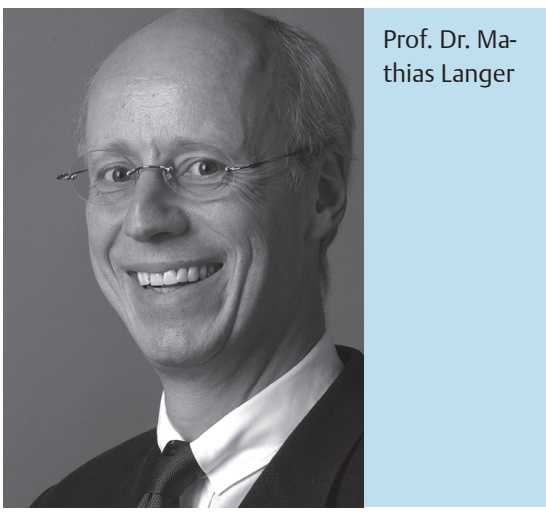

Der diesjährige Deutsche Röntgenkongress findet über Fronleichnam statt:

\section{Mai bis 01. Juni 2013 im CCH in Hamburg.}

Weitere Informationen finden Sie auf www.drg.de 